\title{
Impact of tight glucose control on circulating 3-hydroxybutyrate in critically ill patients
}

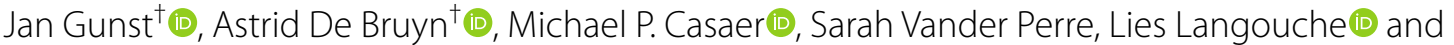 \\ Greet Van den Berghe*
}

\begin{abstract}
Background: Recent evidence suggests a potentially protective effect of increasing ketone body availability via accepting low macronutrient intake early after onset of critical illness. The impact of blood glucose control with insulin on circulating ketones is unclear. Whereas lowering blood glucose may activate ketogenesis, high insulin concentrations may have the opposite effect. We hypothesized that the previously reported protective effects of tight glucose control in critically ill patients receiving early parenteral nutrition may have been mediated in part by activation of ketogenesis.
\end{abstract}

Methods: This is a secondary analysis of 3 randomized controlled trials on tight versus liberal blood glucose control in the intensive care unit, including 700 critically ill children and 2748 critically ill adults. All patients received early parenteral nutrition as part of the contemporary standard of care. Before studying a potential mediator role of circulating ketones in improving outcome, we performed a time course analysis to investigate whether tight glucose control significantly affected ketogenesis and to identify a day of maximal effect, if any. We quantified plasma/serum 3-hydroxybutyrate concentrations from intensive care unit admission until day 3 in 2 matched subsets of 100 critically ill children and 100 critically ill adults. Univariable differences between groups were investigated by Kruskal-Wallis test. Differences in 3-hydroxybutyrate concentrations between study days were investigated by Wilcoxon signed-rank test.

Results: In critically ill children and adults receiving early parenteral nutrition, tight glucose control, as compared with liberal glucose control, lowered mean morning blood glucose on days $1-3(P<0.0001)$ via infusing insulin at a higher dose $(P<0.0001)$. Throughout the study period, caloric intake was not different between groups. In both children and adults, tight glucose control did not affect 3-hydroxybutyrate concentrations, which were suppressed on ICU days 1-3 and significantly lower than the ICU admission values for both groups $(P<0.0001)$.

Conclusion: Tight versus liberal glucose control in the context of early parenteral nutrition did not affect 3-hydroxybutyrate concentrations in critically ill patients. Hence, the protective effects of tight glucose control in this context cannot be attributed to increased ketone body availability.

Keywords: Tight glucose control, Hyperglycemia, Insulin, Critical illness, Ketogenesis, Ketone body, Early parenteral nutrition

\footnotetext{
*Correspondence: greet.vandenberghe@kuleuven.be

${ }^{\dagger} J a n$ Gunst and Astrid De Bruyn have contributed equally to this work. Clinical Division and Laboratory of Intensive Care Medicine, Department of Cellular and Molecular Medicine, KU Leuven, 3000 Leuven, Belgium
}

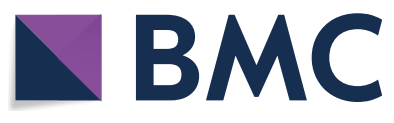

Ther (s) 2021. Open Access This article is licensed under a Creative Commons Attribution 4.0 International License, which permits use, sharing, adaptation, distribution and reproduction in any medium or format, as long as you give appropriate credit to the original author(s) and the source, provide a link to the Creative Commons licence, and indicate if changes were made. The images or other third party material in this article are included in the article's Creative Commons licence, unless indicated otherwise in a credit line to the material. If material is not included in the article's Creative Commons licence and your intended use is not permitted by statutory regulation or exceeds the permitted use, you will need to obtain permission directly from the copyright holder. To view a copy of this licence, visit http://creativecommons.org/licenses/by/4.0/. The Creative Commons Public Domain Dedication waiver (http://creativeco mmons.org/publicdomain/zero/1.0/) applies to the data made available in this article, unless otherwise stated in a credit line to the data. 


\section{Introduction}

Increasing evidence suggests a potentially protective effect of enhancing ketone availability during critical illness [1]. Whereas ketone bodies may serve as vital and more energy-efficient energy substrate than glucose or fatty acids, ketones may also exert signaling functions leading to anti-inflammatory effects and activation of recovery processes such as muscle regeneration and autophagy [2]. In line with this, studies performed in animal models of sepsis and brain injury have demonstrated protective effects of providing ketones or ketogenic diets $[3,4]$. However, human evidence remains scarce, with only a few small randomized controlled trials (RCTs) suggesting improved blood glucose control by ketone supplementation or ketogenic diets [1].

Although ketogenesis has traditionally been reported to be blunted in critical illness [5-7], a recent pilot crossover RCT revealed that four hours of full fasting significantly increased blood ketone concentrations in long-stay critically ill patients [8]. Moreover, we recently demonstrated that withholding parenteral nutrition during the first week of intensive care unit (ICU) stay and hereby temporarily accepting insufficient enteral nutrition, significantly increased ketogenesis in critically ill children and adults $[9,10]$. Activation of ketogenesis by such virtual fasting early during critical illness was most pronounced in children, in whom increased ketone availability also statistically mediated part of the outcome benefit of the intervention [9].

A second metabolic intervention that may affect ketone availability during critical illness is tight glucose control with insulin therapy $[6,11]$, although the net impact of the intervention on ketone concentrations has not been well documented. Indeed, although insulin is a known suppressor of ketogenesis [11-13], critical illness is characterized by profound insulin resistance in the liver-a major site of ketone production-, and in contrast to peripheral insulin resistance, hepatic insulin resistance is difficult to overcome with insulin therapy [14]. Moreover, elevated glucose concentrations may suppress lipolysis and subsequent ketogenesis, whereas lowering blood glucose concentrations could reduce suppression of ketogenesis $[6,11]$. In this regard, a pilot crossover RCT in critically ill adults found activated ketogenesis by lowering blood glucose with insulin therapy [15]. However, in this crossover study, patients received significantly less feeding in periods on tight glucose control, which may have confounded the results. Hence, these findings require confirmation by larger RCTs with equal nutritional intake in both groups.

Our research group previously demonstrated that lowering blood glucose concentrations to the healthy, ageadjusted fasting range significantly improved morbidity and mortality of critically ill children and adults receiving early parenteral nutrition, as compared to tolerating stress hyperglycemia [16-18]. We hypothesize that part of the outcome benefit with tight glucose control in this context may have been mediated by increasing availability of ketone bodies through a mechanism primarily driven by lowering glucose concentrations in the setting of hepatic insulin resistance. We investigated this hypothesis in a secondary analysis of the original RCTs.

\section{Methods}

\section{Patients and study design}

This is a secondary analysis of 3 single-center RCTs performed in the adult surgical, adult medical (NCT00115479) and pediatric ICU (NCT00214916) in Leuven, Belgium, which demonstrated improved clinical outcome by tight glucose control in the ICU [16-18]. The study was performed in accordance with the 1964 Declaration of Helsinki and later amendments, and written informed consent was obtained from the patient or next of kin, or (for children) from the parents or legal guardian. The detailed study protocols and the effect on the primary outcome have been published [16-18]. In brief, patients were randomized upon ICU admission to target age-adjusted normal fasting blood glucose concentrations $(50-80 \mathrm{mg} / \mathrm{dL}[2.8-4.4 \mathrm{mmol} / \mathrm{L}]$ for neonates and infants, $70-100 \mathrm{mg} / \mathrm{dL}$ [3.9-5.6 mmol/L] for children older than 1 year, $80-110 \mathrm{mg} / \mathrm{dL}$ [4.4-6.1 mmol/L] for adults) with insulin therapy (tight glucose control), or to liberal blood glucose control, whereby stress hyperglycemia was only treated when it exceeded $215 \mathrm{mg} /$ $\mathrm{dL}(11.9 \mathrm{mmol} / \mathrm{L})$. All patients received early parenteral nutrition to supplement insufficient or failing enteral nutrition as part of the contemporary standard of care. In all patients, blood samples were taken upon ICU admission, and thereafter daily at $6 \pm 2 \mathrm{~h}$ am. In patients included in the adult medical ICU $(N=1200)$ and pediatric ICU study $(N=700)$, plasma and serum samples were collected. In the adult surgical ICU study $(N=1548)$, only serum samples were collected. After aliquoting, samples were stored at $-80^{\circ} \mathrm{C}$ until further analysis.

In this secondary analysis, we aimed to study whether enhanced ketogenesis could have mediated part of the outcome benefit of tight glucose control seen in the parent RCTs [16-18]. Before studying such potential mediator role through multivariable regression analysis, we performed a time course analysis in 2 matched subsets of critically ill children and adults, to study whether tight glucose control significantly affected ketogenesis in these patients, and to identify a day of maximal effect (if any). To that purpose, we quantified daily circulating 3-hydroxybutyrate (3HB) concentrations from ICU admission until day 3 in the ICU. Patients were eligible if 
they required intensive care for least 3 days, did not have a history of diabetes mellitus and had available blood samples at each time point (upon admission and the 3 consecutive mornings). To rule out selection bias due to an impact of the intervention on ICU length of stay, and for feasibility reasons, the eligible pediatric $(N=274)$, adult medical $(N=537)$ and adult surgical $(N=655)$ patient cohorts were reduced with propensity score matching to obtain 2 matched cohorts of 100 adult and 100 pediatric patients (50 allocated to tight glucose control and 50 allocated to liberal glucose control, of whom (for the adult cohort) 40 from the medical ICU study, and 60 from the surgical ICU study, to obtain a proportional sample from the respective parent RCTs) (Fig. 1). The groups were matched for demographics (age and sex; weight of children, body mass index of adults), baseline type and severity of illness (Pediatric Logistic Organ Dysfunction (PELOD) score for children, Acute Physiology and Chronic Health Evaluation II (APACHE-II) score for adults), need of extracorporeal membrane oxygenation or mechanical hemodynamic support upon admission (for children) and history of malignancy (for adults).

\section{Laboratory analyses}

Blood glucose was measured in whole blood by a blood gas analyzer (ABL700, Radiometer Medical, Copenhagen,
Denmark). The concentration of $3 \mathrm{HB}$ was quantified with a laboratory assay based upon the oxidation of $3 \mathrm{HB}$ to acetoacetate by the enzyme 3HB dehydrogenase and the concomitant reduction of $\mathrm{NAD}^{+}$to $\mathrm{NADH}$, as previously described for plasma samples $[9,19]$. Samples with undetectable $3 \mathrm{HB}$ concentrations were assigned the detection limit $(0.04 \mathrm{mmol} / \mathrm{L})$. In the pediatric cohort, $3 \mathrm{HB}$ was determined in plasma samples. As plasma was not available for the adult surgical ICU study, we first evaluated the validity of serum as a replacement for plasma by performing $3 \mathrm{HB}$ measurements in paired samples of adult patients included in the medical ICU study (40 patients with 4 paired samples, leading to 160 paired measurements). The mean difference of these paired measurements was $0.015 \mathrm{mmol} / \mathrm{L}$ (95\% confidence interval ranging from -0.117 to $0.133 \mathrm{mmol} / \mathrm{L}$ ). Since this confirmed validity of serum as replacement for plasma, $3 \mathrm{HB}$ was subsequently quantified in all serum samples of the adult cohort.

\section{Statistical analyses}

Data are presented as frequencies and percentages or as medians with interquartile range. Fisher's exact test and Kruskal-Wallis test were used to analyze univariable differences between patient groups, as appropriate. Wilcoxon signed-rank test was used to analyze paired

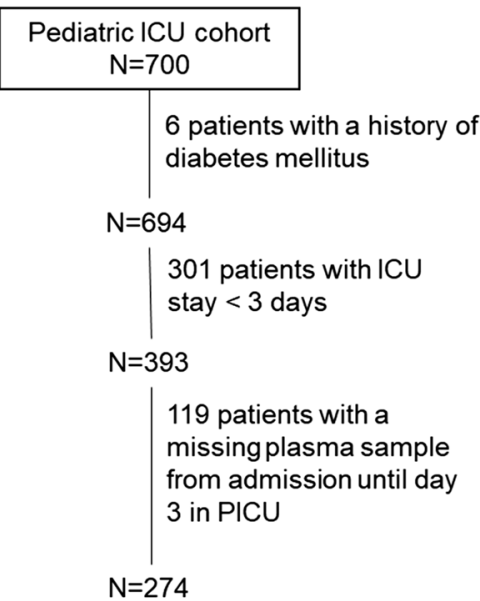

$\mathrm{N}=274$

Matching for age, weight, sex, diagnostic category, severity of illness* ${ }^{*} \mathrm{ECMO}$ or other assist device

$\mathrm{N}=100$
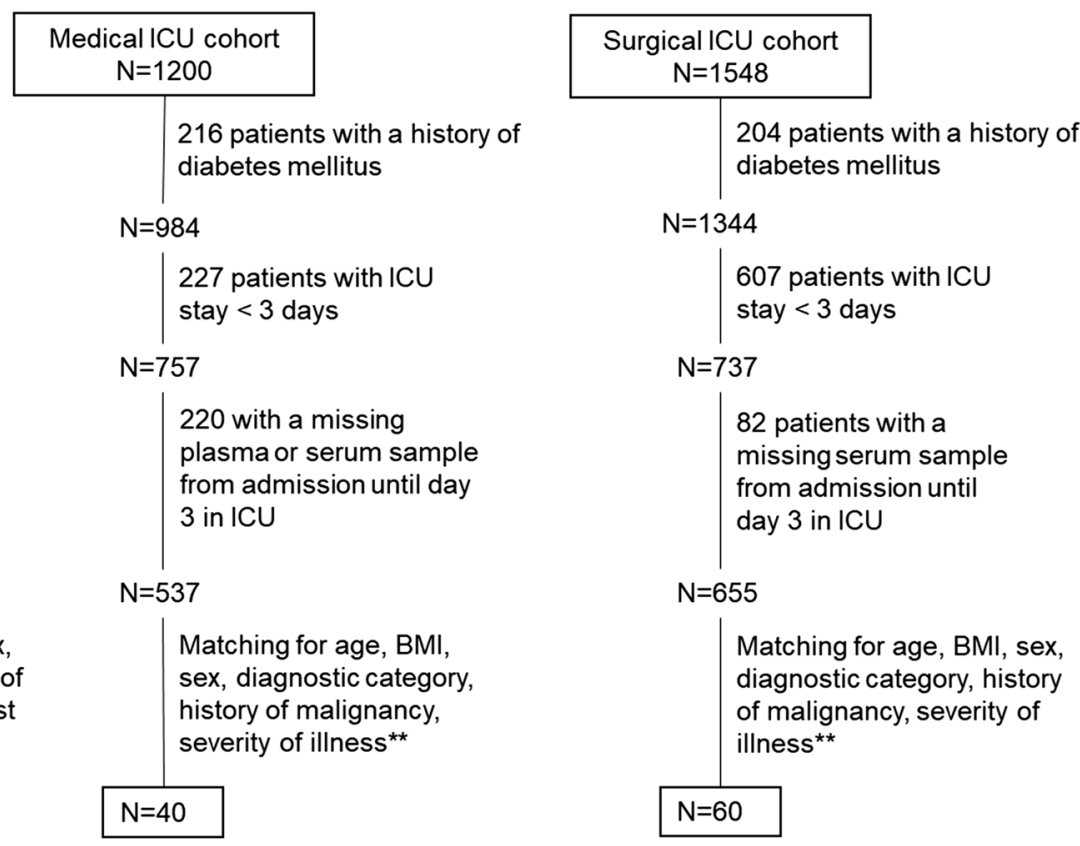

*As evaluated by the PELOD score (=PEdiatric Logistic Organ Dysfunction score) first $24 \mathrm{~h}$ in PICU

** As evaluated by the APACHE-II score (=Acute Physology and Chronic Health Evaluation) first $24 \mathrm{~h}$ in ICU

Fig. 1 Selection and matching 
differences in ketone concentrations between study days. All analyses were performed with the use of JMP software, version pro 15 (SAS Institute, NC, USA). Statistical significance was set at a $P$ value of 0.05 .

\section{Results}

Effect of tight versus liberal glucose control on plasma 3HB concentrations in critically ill children

The matched cohort of critically ill children consisted of 50 patients randomized to tight glucose control and 50 patients randomized to liberal glucose control (Fig. 1). Baseline characteristics were similar between both groups (Table 1). Upon admission, blood glucose was comparable between both groups $(P=0.08)$, after which morning blood glucose was significantly lower in tight glucose control patients on all study days (all $P<0.0001$ ) (Fig. 2a), in the presence of higher insulin doses (all $P<0.0001$ ) (Fig. 2b) and similar intake through enteral and parenteral nutrition (Fig. 2c). From admission onward, plasma $3 \mathrm{HB}$ concentrations were similarly low in both randomization groups (Fig. 2d), with a significant decrease in concentrations between ICU admission and day $1(P<0.0001)$. Of all $4003 \mathrm{HB}$ determinations, concomitant hypoglycemia $(\leq 40 \mathrm{mg} /$ $\mathrm{dL}[\leq 2.2 \mathrm{mmol} / \mathrm{L}])$ on the corresponding blood glucose measurement was present at 5 occasions ( 1 blood glucose measurement upon randomization in each randomization group, thereafter 1 measurement at each time point in the tight glucose control group).

\section{Effect of tight versus liberal glucose control on serum 3HB concentrations in critically ill adults}

The matched cohort of critically ill adults consisted of 100 patients -50 patients per randomization group-of whom 60 were admitted to the surgical ICU and 40 to the medical ICU (Fig. 1). Baseline characteristics were similar (Table 2, Additional file 1: Table S1). As was the case in the total adult study population [20], ICU mortality was significantly lower in tight glucose control patients $(P=0.01)$. Blood glucose concentrations were not different upon ICU admission $(P=0.6)$ and were significantly lower in tight glucose control patients throughout the first 3 days in ICU (all $P<0.0001$ ) (Fig. 3a), through infusion of higher insulin doses (all $P<0.0001$ ) (Fig. $3 \mathrm{~b}$ ) and in the presence of similar caloric intake (Fig. 3c). In both study groups, serum $3 \mathrm{HB}$ was similarly suppressed from days 1 to 3 (Fig. 3d), with a significant decrease from ICU admission to day $1(P<0.0001)$. Of all $4003 \mathrm{HB}$ determinations, concomitant hypoglycemia was present on one corresponding

Table 1 Baseline characteristics and outcome of matched children

\begin{tabular}{|c|c|c|c|}
\hline Baseline characteristics & Liberal GC $(N=50)$ & Tight GC $(N=50)$ & $P$ value \\
\hline Age (years)—median (IQR) & $0.9(0.2-2.9)$ & $0.6(0.2-3.1)$ & 0.79 \\
\hline Age group ${ }^{a}$ & & & 0.39 \\
\hline Neonate-no. (\%) & $4(8)$ & $8(16)$ & \\
\hline Infant—no. (\%) & $23(46)$ & $19(38)$ & \\
\hline Child—no. (\%) & $23(46)$ & $23(46)$ & \\
\hline Weight (kg)—median (IQR) & $7.7(4.2-11.5)$ & $6.6(3.8-13.9)$ & 0.75 \\
\hline Sex (male)—no. (\%) & $25(50)$ & $27(54)$ & 0.84 \\
\hline Diagnostic category & & & 1.00 \\
\hline \multicolumn{4}{|l|}{ Surgical } \\
\hline Transplantation—no. (\%) & $1(2)$ & $1(2)$ & \\
\hline Cardiac surgery-no. (\%) & $41(82)$ & $40(80)$ & \\
\hline Other surgery—no. (\%) & $4(8)$ & $5(10)$ & \\
\hline Medical一no. (\%) & $4(8)$ & $4(8)$ & \\
\hline PELOD first $24 \mathrm{~h}$ in ICU—median (IQR) & $12(11-12)$ & $12(11-12)$ & 0.53 \\
\hline ECMO or other assist device-no. (\%) & $1(2)$ & $1(2)$ & 1.00 \\
\hline \multicolumn{4}{|l|}{ Outcome } \\
\hline ICU mortality—no. (\%) & $4(8)$ & $1(2)$ & 0.36 \\
\hline Length of ICU stay (days) — median (IQR) & $5(3-9)$ & $6(3-10)$ & 0.80 \\
\hline Patients with secondary infection—no. (\%) & $28(56)$ & $23(46)$ & 0.42 \\
\hline
\end{tabular}

a Neonate corresponds to children aged $<4$ weeks, Infants to children aged between 4 weeks and 1 year, Child to children aged $>1$ year. BMI is body mass index, PELOD is Pediatric Logistic Organ Dysfunction score, ECMO is extracorporeal membrane oxygenation, GC is glucose control, IQR is interquartile range 
A.

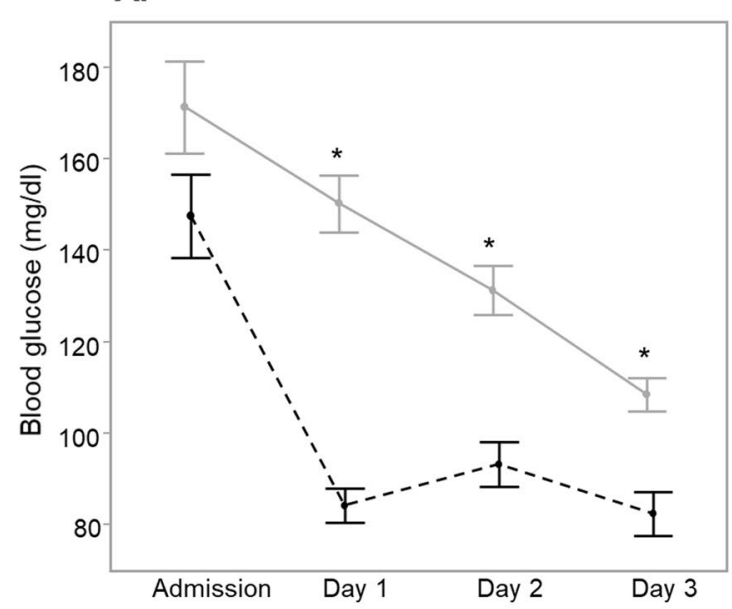

C.

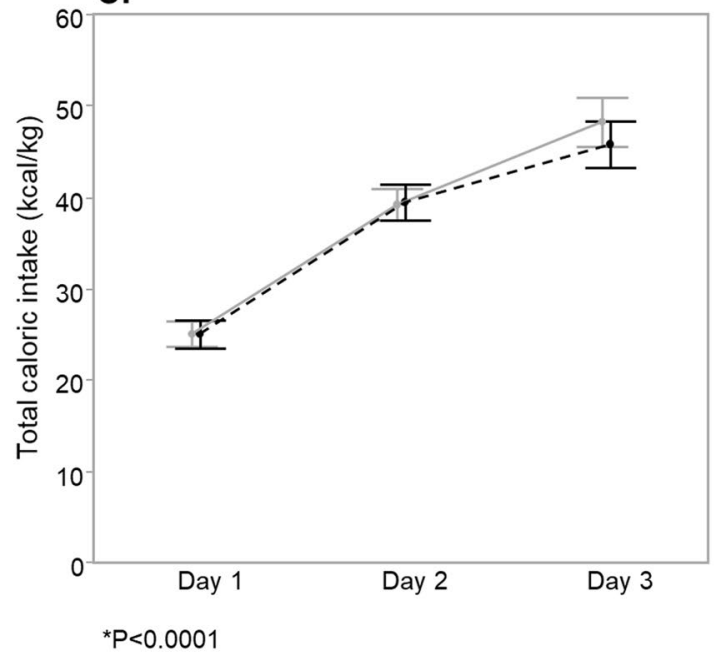

B.

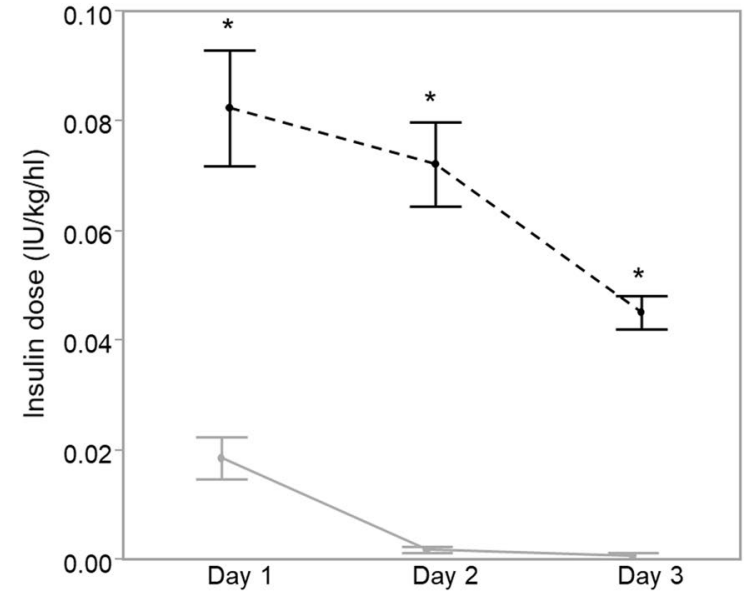

D.

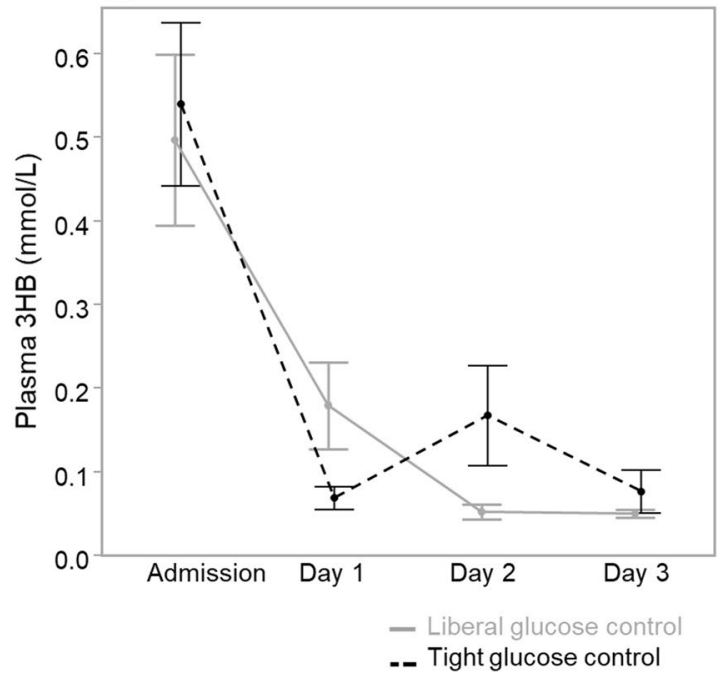

Fig. 2 Admission and morning blood glucose concentrations (a), insulin dose (b), total caloric intake through enteral and parenteral nutrition (c) and plasma 3-hydroxybutyrate (3HB) concentrations (d) during the first 3 days in the ICU in a matched subset of critically ill children randomized to tight (dotted black line) or liberal (full gray line) glucose control. Data are shown as mean and standard error. Samples with undetectable $3 \mathrm{HB}$ concentrations were assigned the detection limit $(0.04 \mathrm{mmol} / \mathrm{L}): 47$ upon ICU admission ( 23 and 24 in tight and liberal group, respectively), 84 at day 1 (45 and 39 in tight and liberal group, respectively), 91 at day 2 ( 43 and 48 in tight and liberal group, respectively) and 92 at day 3 (46 in each group). ${ }^{*} P \leq 0.0001$. For conversion of blood glucose in $\mathrm{mg} / \mathrm{dL}$ to $\mathrm{mmol} / \mathrm{L}$, divide by 18

blood glucose measurement at day 1 in a patient receiving liberal glucose control.

\section{Discussion}

In this secondary analysis of three RCTs on the impact of tight glucose control in critically ill children and adults receiving early parenteral nutrition, we found no impact of the intervention on circulating ketone concentrations. Indeed, regardless of randomization, $3 \mathrm{HB}$ concentrations decreased from ICU admission until day 1 to very low levels and remained suppressed until day 3 in ICU. These data suggest that the protective effects of tight glucose control in these RCTs were not mediated by increased ketone availability.

The data contrast with a previous pilot crossover RCT performed in critically ill adults, which found increased ketone concentrations by tight glucose control [15]. However, in this crossover RCT, patients received significantly less nutritional intake while receiving tight glucose control, which may have confounded the results. In the current study, nutritional intake was comparable in tight and liberal glucose control patients, as all patients received 
Table 2 Baseline characteristics and outcome of matched adults

\begin{tabular}{|c|c|c|c|}
\hline Baseline characteristics & Liberal GC $(N=50)$ & Tight GC $(N=50)$ & $P$ Value \\
\hline Age (years) — median (IQR) & $65(56-74)$ & $66(55-74)$ & 0.73 \\
\hline Length (cm)—median (IQR) & $170(165-176)$ & $172(170-179)$ & 0.27 \\
\hline BMI $\left(\mathrm{kg} / \mathrm{m}^{2}\right)$-median (IQR) & $24.2(21.6-27.7)$ & $24.8(22.0-27.5)$ & 0.53 \\
\hline Sex (male)—no. (\%) & $41(82)$ & $45(90)$ & 0.39 \\
\hline Diagnostic group—no. (\%) & & & 0.93 \\
\hline \multicolumn{4}{|l|}{ Surgical } \\
\hline Transplantation—no. (\%) & $4(8)$ & $2(4)$ & \\
\hline Cardiac surgery-no. (\%) & $17(34)$ & $19(38)$ & \\
\hline Other surgery—no. (\%) & $9(18)$ & $9(18)$ & \\
\hline Medical—no. (\%) & $20(40)$ & $20(40)$ & \\
\hline History of malignancy—no. (\%) & $6(12)$ & $6(12)$ & 1.00 \\
\hline APACHE-II score-median (IQR) & $12(8-19)$ & $12(8-21)$ & 0.93 \\
\hline \multicolumn{4}{|l|}{ Outcome } \\
\hline ICU mortality—no. (\%) & $16(32)$ & $5(10)$ & 0.01 \\
\hline Length of ICU stay (days)—-median (IQR) & $8(4-18)$ & $6(4-14)$ & 0.33 \\
\hline
\end{tabular}

BMI is body mass index, APACHE-II is Acute Physiology and Chronic Health Evaluation II, IQR is interquartile range, GC is glucose control

early parenteral nutrition as part of the contemporary standard of care. Interestingly, the observed suppression of ketone concentrations in both critically ill children and adults from day 1 in ICU onward mirrors the suppression observed earlier by providing early parenteral nutrition as compared with no parenteral nutrition during the first week in the ICU $[9,10]$. The powerful suppression of ketogenesis by providing early full nutritional support may be difficult to counter by lowering glucose concentrations with insulin. It currently remains unclear whether tight glucose control in the absence of early parenteral nutrition affects ketogenesis, and whether this is associated with clinical benefit, which needs to be investigated [21]. Nevertheless, the current study suggests that the protective effects of tight glucose control in the context of providing early parenteral nutrition are mediated by other pathways than activation of ketogenesis. In this regard, previous studies have put forward prevention of intracellular glucose toxicity and hereby avoidance of mitochondrial damage in vital organs as potentially protective pathways activated by blood glucose lowering [22].

As in previous studies, upon ICU admission $3 \mathrm{HB}$ concentrations were higher in children than in adults $[9,10]$. Also in health, the ketogenic response is known to be more pronounced in children [23]. Although we do not have data on pre-admission nutritional intake, most patients were presumably fasted prior to ICU admission. Hence, the significant decline in $3 \mathrm{HB}$ concentrations soon after ICU admission is likely explained by the initiation of early nutritional support. Alternatively, ketogenesis could be suppressed by even small doses of insulin initiated after ICU admission [23], also in liberal glucose control patients. However, in such case, one would have expected a less powerful or temporary suppression of ketogenesis in patients receiving liberal glucose control, since the administered insulin dose was significantly lower, with a considerable number of patients requiring no or only temporary insulin treatment.

This study was performed on prospectively collected samples obtained in the context of 3 large RCTs that showed benefit of tight glucose control in the ICU, which is a strength. This study inherently also has limitations. For feasibility reasons and due to missing samples, we restricted the analyses to a subset of patients at four time points. However, patients were well matched for baseline characteristics, and the outcome differences in the adult cohort mimicked the difference in the total cohort [20], suggesting the subsets are representative for the total study population. Moreover, the consistent results in both critically ill adults and children corroborate our findings. Nevertheless, we cannot exclude the possibility that the subset of patients is not fully representative for the total study population, or that the intervention may have affected ketone concentrations at other time points. Second, we cannot exclude potential bias by prolonged storage of samples. However, all samples were stored at $-80{ }^{\circ} \mathrm{C}$, and the measured range of ketone concentrations was comparable to the obtained range in more recent patient studies $[9,10]$. Moreover, we previously found no impact of a freeze-thaw cycle and short-term storage at ambient temperature on $3 \mathrm{HB}$ measurements [24]. Hence, we 
A.

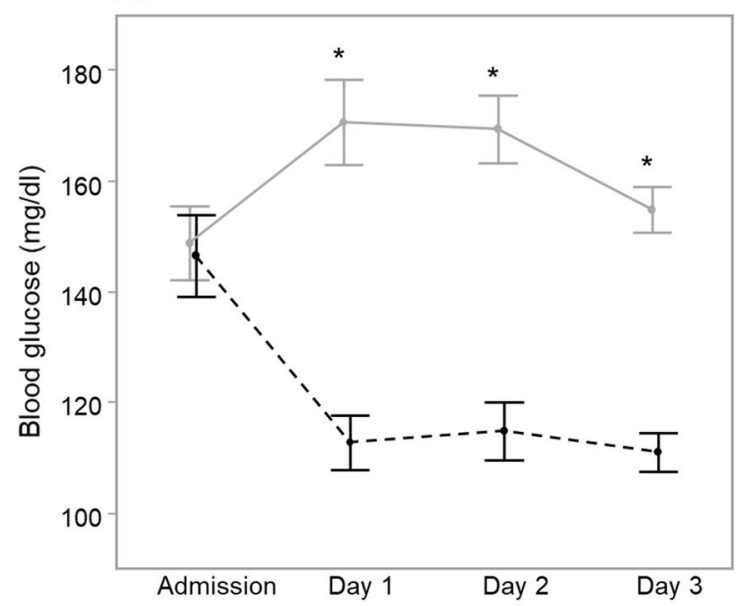

C.

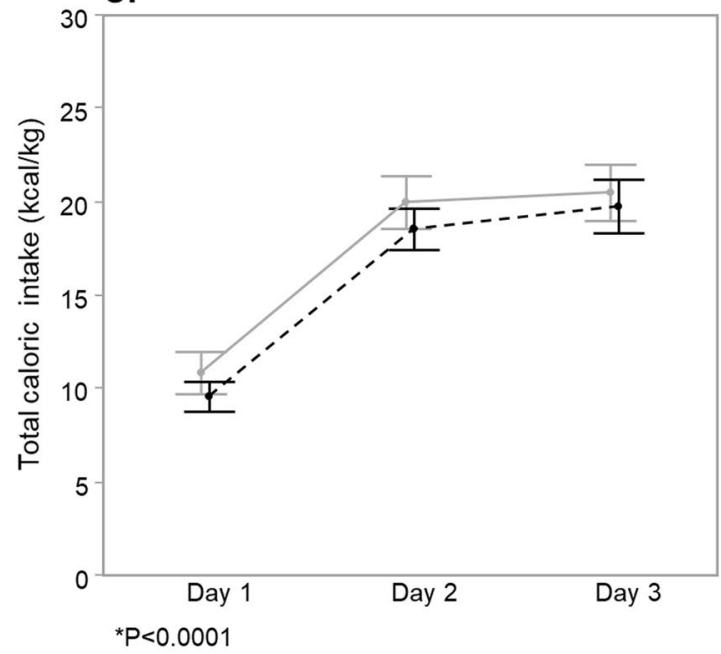

B.

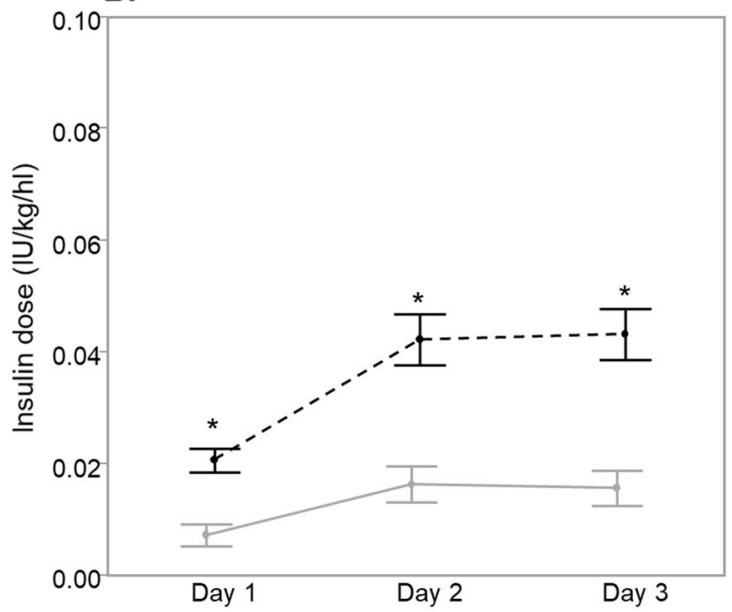

D.

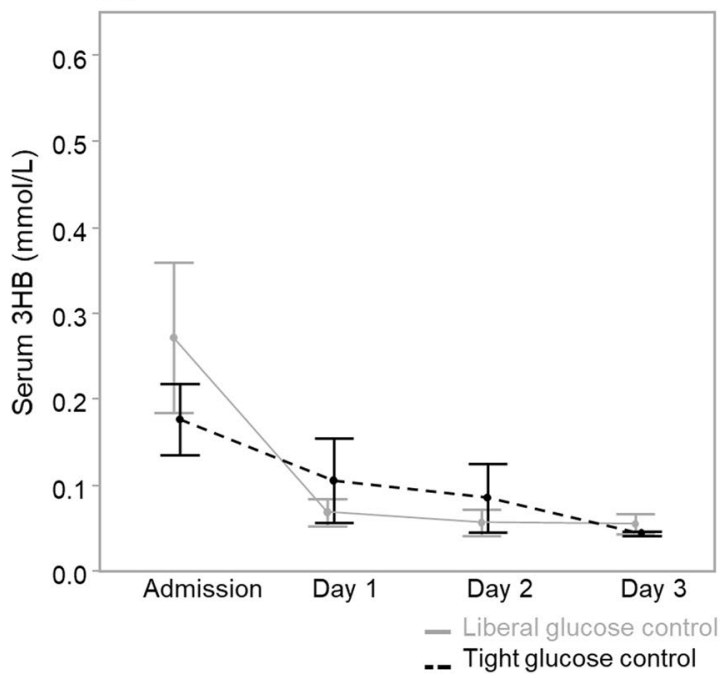

Fig. 3 Admission and morning blood glucose concentrations (a), insulin dose (b), total caloric intake through enteral and parenteral nutrition (c) and serum 3-hydroxybutyrate (3HB) concentrations (d) during the first 3 days in the ICU in a matched subset of critically ill adults randomized to tight (dotted black line) or liberal (full gray line) glucose control. Data are shown as mean and standard error. Samples with undetectable $3 \mathrm{HB}$ concentrations were assigned the detection limit $(0.04 \mathrm{mmol} / \mathrm{L}): 69$ upon ICU admission (34 and 35 in tight and liberal group, respectively), 92 at day 1 (46 in each group), 94 at day 2 (46 and 48 in tight and liberal group, respectively) and 95 at day 3 (48 and 47 in tight and liberal group, respectively). ${ }^{*} P \leq 0.0001$. For conversion of blood glucose in $\mathrm{mg} / \mathrm{dL}$ to $\mathrm{mmol} / \mathrm{L}$, divide by 18

consider artifacts induced by prolonged cold storage unlikely. Third, since a considerable number of samples had results for $3 \mathrm{HB}$ below the detection limit of the assay, we cannot exclude a minor effect of the intervention on ketogenesis. However, if present, such a small effect would likely be clinically irrelevant.

\section{Conclusion}

Tight glucose control did not significantly affect circulating $3 \mathrm{HB}$ concentrations in critically ill children and adults receiving early parenteral nutrition. These data suggest that the protective effects of tight glucose control in this context could not be attributed to increased ketone body availability.

\section{Supplementary Information}

The online version contains supplementary material available at https://doi. org/10.1186/s13054-021-03772-6.

Additional file 1. Table S1 Baseline characteristics and outcome of matched adults 


\section{Acknowledgements \\ Not applicable.}

\section{Authors' contributions}

$J G, A D B, L L, M P C$ and GVdB conceived and designed the experiments. SVP performed the laboratory analyses. ADB, JG, LL and GVdB analyzed the data. ADB and JG wrote the first draft, which was subsequently revised by all authors. All authors read and approved the final manuscript.

\section{Funding}

This work was supported by an ERC Advanced Grant from the European Union's Horizon 2020 Programme to GVdB (AdvG-2017-785809); by the Methusalem programme of the Flemish Government (through the University of Leuven) to GVdB and LL (METH14/06); by the Agency for Innovation through Science and Technology, Flanders, Belgium to GVdB (IWT/070695/ TBM); by the Research Foundation Flanders (FWO), Belgium (to LL and GVdB, G.0C78.17N; postdoctoral research fellowship to MPC); by the University Hospitals Leuven (postdoctoral research fellowship by the Clinical Research and Education Council to JG)

\section{Availability of data and materials}

Data sharing will be considered under the form of collaborative projects. Proposals can be directed to the corresponding author.

\section{Declarations}

\section{Ethics approval and consent to participate}

This is a secondary analysis of 3 monocenter (Leuven, BE) randomized controlled trials (ML 2586 and ClinicalTrials.gov NCT00214916, $n=700$ ML1820 and ClinicalTrials.gov NCT00115479, $n=1200 ;$ ML1094, $n=1548$ ). The institutional review board approved the study protocols. Written informed consent was obtained from the patients or next of kin, or (for children) from the parents or legal guardian.

\section{Consent for publication}

Not applicable.

\section{Competing interests}

We declare no competing interests.

Received: 7 July 2021 Accepted: 14 September 2021

Published online: 25 October 2021

\section{References}

1. Gunst J, Casaer MP, Langouche L, Van den Berghe G. Role of ketones, ketogenic diets and intermittent fasting in ICU. Curr Opin Crit Care. 2021;27(4):385-9.

2. Newman JC, Verdin E. Ketone bodies as signaling metabolites. Trends Endocrinol Metab. 2014;25(1):42-52

3. Goossens C, Weckx R, Derde S, Dufour T, Vander Perre S, Pauwels L, et al. Adipose tissue protects against sepsis-induced muscle weakness in mice: from lipolysis to ketones. Crit Care. 2019;23(1):236.

4. White H, Venkatesh B. Clinical review: ketones and brain injury. Crit Care. 2011;15(2):219.

5. Beisel WR, Wannemacher RW Jr. Gluconeogenesis, ureagenesis, and ketogenesis during sepsis. JPEN J Parenter Enteral Nutr. 1980;4(3):277-85.

6. Beylot M, Guiraud M, Grau G, Bouletreau P. Regulation of ketone body flux in septic patients. Am J Physiol. 1989;257(5 Pt 1):E665-74.
7. Birkhahn RH, Long CL, Fitkin DL, Busnardo AC, Geiger JW, Blakemore WS. A comparison of the effects of skeletal trauma and surgery on the ketosis of starvation in man. J Trauma. 1981:21(7):513-9.

8. Van Dyck L, Vanhorebeek I, Wilmer A, Schrijvers A, Derese I, Mebis L, et al. Towards a fasting-mimicking diet for critically ill patients: the pilot randomized crossover ICU-FM-1 study. Crit Care. 2020;24(1):249.

9. De Bruyn A, Gunst J, Goossens C, Vander Perre S, Guerra GG, Verbruggen $\mathrm{S}$, et al. Effect of withholding early parenteral nutrition in PICU on ketogenesis as potential mediator of its outcome benefit. Crit Care. 2020;24(1):536

10. De Bruyn A, Langouche L, Vander Perre S, Gunst J, Van den Berghe G. Impact of withholding early parenteral nutrition in adult critically ill patients on ketogenesis in relation to outcome. Crit Care. 2021;25(1):102.

11. Alberti KG, Johnston DG, Gill A, Barnes AJ, Orskov H. Hormonal regulation of ketone-body metabolism in man. Biochem Soc Symp. 1978;43:163-82.

12. Beylot M, Picard S, Chambrier C, Vidal H, Laville M, Cohen R, et al. Effect of physiological concentrations of insulin and glucagon on the relationship between nonesterified fatty acids availability and ketone body production in humans. Metabolism. 1991;40(11):1138-46.

13. Keller U, Gerber PP, Stauffacher W. Fatty acid-independent inhibition of hepatic ketone body production by insulin in humans. Am J Physiol. 1988;254(6 Pt 1):E694-9.

14. Langouche L, Vander Perre S, Wouters PJ, D'Hoore A, Hansen TK, Van den Berghe $\mathrm{G}$. Effect of intensive insulin therapy on insulin sensitivity in the critically ill. J Clin Endocrinol Metab. 2007;92(10):3890-7.

15. Wolahan SM, Prins ML, McArthur DL, Real CR, Hovda DA, Martin NA, et al. Influence of glycemic control on endogenous circulating ketone concentrations in adults following traumatic brain injury. Neurocrit Care. 2017;26(2):239-46.

16. Van den Berghe G, Wilmer A, Hermans G, Meersseman W, Wouters PJ, Milants I, et al. Intensive insulin therapy in the medical ICU. N Engl J Med. 2006;354(5):449-61.

17. Van den Berghe $G$, Wouters P, Weekers F, Verwaest C, Bruyninckx F, Schetz $M$, et al. Intensive insulin therapy in critically ill patients. N Engl J Med. 2001;345(19):1359-67.

18. Vlasselaers D, Milants I, Desmet L, Wouters PJ, Vanhorebeek I, van den Heuvel I, et al. Intensive insulin therapy for patients in paediatric intensive care: a prospective, randomised controlled study. Lancet. 2009;373(9663):547-56

19. Williamson DH, Mellanby J, Krebs HA. Enzymic determination of D()-beta-hydroxybutyric acid and acetoacetic acid in blood. Biochem J. 1962;82(1):90-6.

20. Van den Berghe G, Wilmer A, Milants I, Wouters PJ, Bouckaert B, Bruyninckx $F$, et al. Intensive insulin therapy in mixed medical/surgical intensive care units: benefit versus harm. Diabetes. 2006;55(11):3151-9.

21. Gunst J, De Bruyn A, Van den Berghe G. Glucose control in the ICU. Curr Opin Anaesthesiol. 2019;32(2):156-62.

22. Gunst J, Van den Berghe G. Blood glucose control in the ICU: don't throw out the baby with the bathwater! Intensive Care Med. 2016;42(9):1478-81.

23. Cahill GF. Fuel metabolism in starvation. Annu Rev Nutr. 2006;26:1-22.

24. Hendrickx M, Van Dyck L, Vanhorebeek I, Wilmer A, Schrijvers A, Derese I, et al. The impact of ambient temperature and freeze-thaw on ketone measurements. Abstract presented at the Annual Meeting of the Belgian Society of Intensive Care Medicine. 2020 Dec 17. Ref 12/2020.04.

\section{Publisher's Note}

Springer Nature remains neutral with regard to jurisdictional claims in published maps and institutional affiliations. 